\title{
Multifocal low-grade astrocytoma successfully treated with volumetric modulated arc therapy and concurrent temozolomide
}

\begin{abstract}
Summary
Multifocal gliomas comprise an uncommon clinical entity with challenging diagnostic and therapeutic ramifications. In this article, we present the case of a young man with progressive memory loss that was found to have numerous space-occupying lesions masquerading as secondary metastases in his brain MRI. Histopathological examination revealed a diffuse low-grade astrocytoma. The patient was successfully treated with radical chemoradiotherapy and adjuvant temozolomide and has been recurrence-free during three years of regular follow-up. While we review the contemporary diagnostic and therapeutic options, we emphasize the necessity of stereotactic biopsy to 1 . exclude brain metastases, 2 . provide tissue for further molecular characterization, which is helpful for prognostication, and 3. guide an individualized treatment plan. We also contend against using whole-brain radiotherapy based on its well-recognized detrimental neurocognitive effects and its inability to allow dose escalation. Instead, targeted radiotherapy with IMRT/VMAT should be the preferred irradiation technique. As our imaging technology advances, so does our ability to diagnose multifocal lesions that would have been classified as unifocal in the past. Hence, we expect that we will, more often, be finding ourselves confronted with this clinical scenario in the future.
\end{abstract}

Keywords: multifocal astrocytoma, volumetric modulated arc therapy, temozolomide
Volume 12 Issue 6 - 202I

\author{
Efstathios Kamperis, MD, MSc,' Dimitrios \\ Dionysopoulos, MD, $\mathrm{PhD},{ }^{2}$ Konstantinos \\ Efthymiadis, MD, MSc, ${ }^{2}$ Chionia Kodona, \\ MSc, PhD, ${ }^{3}$ Thomas Zarampoukas, MD, PhD, ${ }^{4}$ \\ Konstantinos Hatziioannou, $\mathrm{PhD},{ }^{3}$ Vasileios \\ Giannouzakos, MD' \\ 'Radiation Oncology Department, Papageorgiou General \\ Hospital, Greece \\ 2Medical Oncology Department, Papageorgiou General Hospital, \\ Greece \\ ${ }^{3}$ Medical Physics Department, Papageorgiou General Hospital, \\ Greece \\ ${ }^{4}$ Department of Pathology, School of Medicine, Aristotle \\ University of Thessaloniki, Greece
}

\begin{abstract}
Correspondence: Efstathios Kamperis, Radiation Oncology Department, Papageorgiou General Hospital, Agiou Pavlou 76, PO 564 29, Thessaloniki, Greece, Tel 23 I3 32 34I7, Email ekamperi@gmail.com
\end{abstract}

Received: December 19, 202I | Published: December 27, 2021 


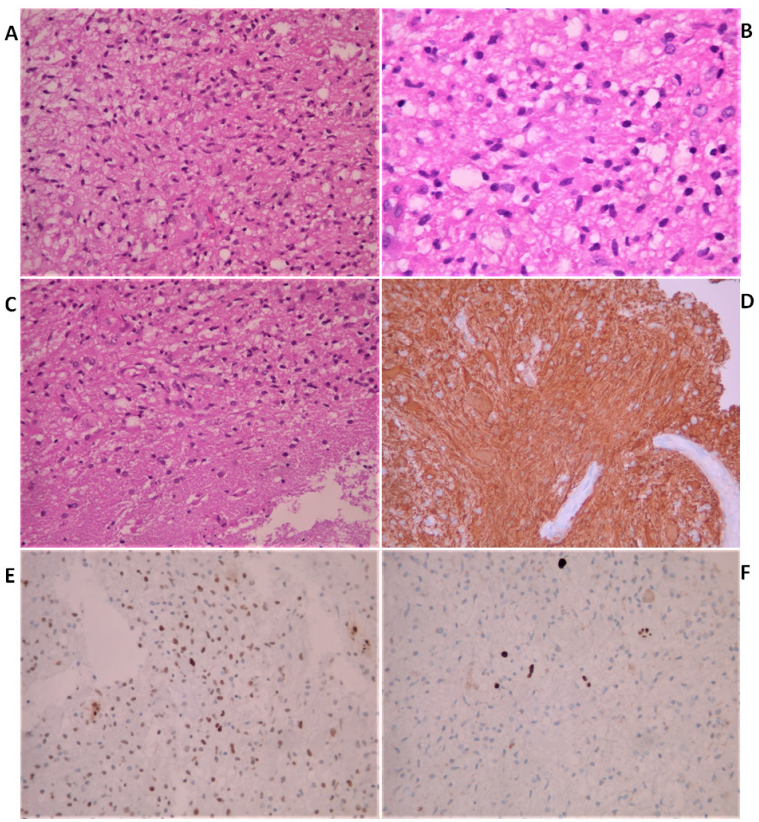

Figure 2 Histological findings of stereotactic biopsy from a brain lesion. A Astrocytoma: Mild to moderate cellularity and mild nuclear atypia. H\&E x200 B. No visible mitosis. H\&E $\times 40 \mathrm{C}$. Invasion of white matter by the astrocytoma. H\&E x200 D. Tumor cells are positive for GFAP. Immunostain x200 E. Many cells show nuclear positivity for $\mathrm{p} 53$. Immunostain $\times 200 \mathrm{~F}$. Few cells show nuclear positivity for Ki67. Immunostain $\times 200$.
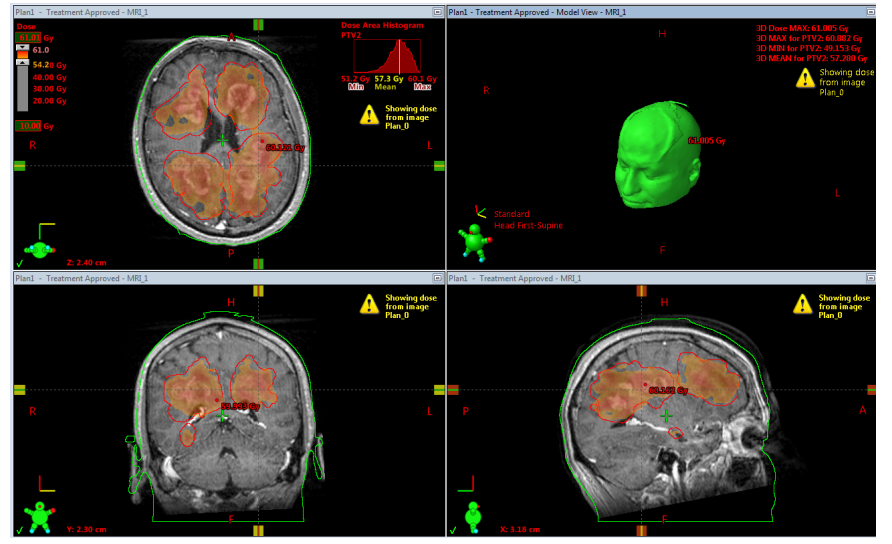

Figure 3 Dose distribution superimposed with brain MRI. The patient was treated with VMAT with a simultaneous integrated boost, receiving 54 Gy in 30 sessions with 1.8 Gy per session to the enhancing lesions of the brain and 50 Gy to the peritumoral edema.

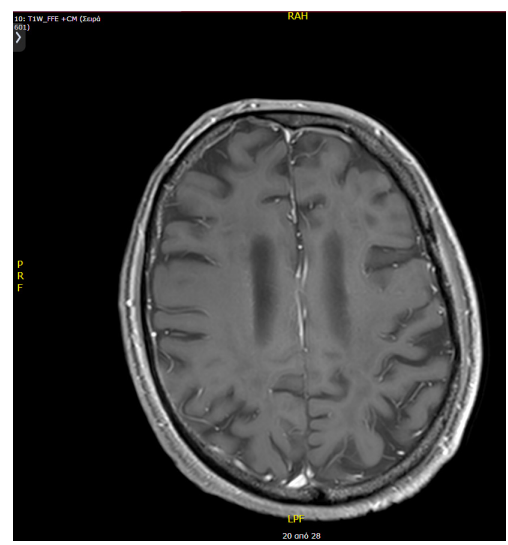

Figure 4 Follow-up brain MRI three years later from the initial diagnosis showing complete radiological response.

\section{Discussion}

Multiple low-grade gliomas are well recognized but relatively under-reported in the literature. Therefore, we often have to extrapolate knowledge from multiple glioblastomas. ${ }^{3}$ Based on their underlying mechanism of development, they are divided into multifocal and multicentric. The former spread via established pathways, e.g., commissural fibers, cerebrospinal fluid channels, blood vessels, or via local extension with satellite formation. ${ }^{4}$ The latter constitute widely separated lesions located in different lobes or hemispheres whose development cannot be explained based on the previously mentioned pathways. Therefore, when multiple lesions are connected via a T2 or fluid-attenuated inversion recovery (FLAIR) high signal in brain MRI, they may be considered multifocal. Conversely, when they are not, they may be regarded as multicentric. ${ }^{2}$ Even in the absence of macroscopic continuity between the lesions, the microscopic study could still reveal areas of continuity. ${ }^{4}$ The clinical significance of the above distinction remains to be determined. ${ }^{5,6}$

The gold-standard imaging modality for the diagnosis and monitoring of low-grade gliomas is MRI. ${ }^{7}$ These tumors typically appear as low-signal lesions on T1-weighted sequences and as high signal on T2-weighted and FLAIR sequences, often located in the eloquent areas of the brain. The lesions are typically non-enhancing, but this is not always the case as in $15-40 \%$ they show some enhancement. $^{8-10}$ This may represent an anaplastic progression of a pre-existing low-grade gliomas, ${ }^{7}$ which the biopsy could easily miss given the heterogeneity of the disease. ${ }^{11}$ On the other hand, up to $1 / 3$ of non-enhancing lesions may represent high-grade gliomas. ${ }^{12}$ Hence, tumor grading does not have a one-to-one correspondence with radiologic phenotype. More advanced MR techniques include MR spectroscopy and perfusion imaging that provide complementary physiological information regarding the tumor's metabolism and haemodynamics. ${ }^{7}$

Multifocal gliomas can easily be misdiagnosed as metastatic tumors, ${ }^{13}$ particularly when the number of lesions is large. Differential diagnosis between gliomas and metastases is, to some extent, possible by MRI. ${ }^{13}$ Concretely, multifocal gliomas are usually located within the deep white matter, as opposed to metastases that tend to center at the gray matter-white matter interface. ${ }^{13,14}$ A CT scan of the chest and abdomen is standard practice, and in the absence of a primary tumor, a stereotactic biopsy will establish the correct diagnosis and provide adequate tissue for further molecular tests. ${ }^{15}$ The differential diagnosis also includes central nervous system lymphoma and inflammatory diseases such as tumefactive multiple sclerosis. ${ }^{16,17}$ Such demyelinating lesions can mimic tumors,${ }^{18}$ and among misdiagnoses, a low-grade astrocytoma is the most frequent. ${ }^{16}$

The underlying pathogenetic mechanism for multiple gliomas has been both an intriguing and unsolved issue. There are three proposed theories. ${ }^{14}$ Tumor cells may spread along white matter tracts or cerebrospinal fluid to distant locations. The subventricular zone, in particular, has been known to shelter cells with enhanced proliferative capacity and has been described as an area generating multiple and multicentric GBM via the expression of metalloproteinase and mesenchymal markers. ${ }^{2}$ The second theory suggests that, sporadically, a large area of the brain infiltrated by a diffuse low-grade astrocytoma acts as a field where malignant transformation occurs, engendering higher grade gliomas. The underlying diffuse astrocytoma may evade imaging's resolution, but several distinct foci of ring-enhancing lesions will be seen suggestive of a high-grade tumor, like in our case. Last, a patient harboring some genetic abnormality may be predisposed to the development of multiple lesions without the need for pre-existing diffuse dissemination of the disease. The majority of 
brain tumors in adults are sporadic in the sense that no risk factor can be identified. Besides some relatively rare genetic syndromes, e.g., neurofibromatosis, Li-Fraumeni, the only known risk factor for primary brain tumors is ionizing radiation. ${ }^{19}$

Low-grade gliomas tend to grow diffusely into the normal brain, and epileptic seizures are their archetypal symptomatic expression. ${ }^{20}$ Moreover, among the various subtypes, diffuse low-grade gliomas are the most epileptogenic. ${ }^{21}$ Our patient had high volume disease; nevertheless, he had no history of seizures or other neurological symptoms besides memory loss. In other cases, the accompanying cerebral edema or the blockage of normal cerebrospinal circulation may be the culprit for the symptomatic manifestation of the disease. Non specific symptoms can include headache, nausea and vomiting, altered consciousness/mental state, whereas specific symptoms depend on the affected area of the brain.

The 2016 WHO classification system now classifies diffuse astrocytomas based on isocitrate dehydrogenase (IDH) mutation status and several other critical molecular genetic alterations (ATRX mutations, TP53 mutations, H3K27M mutations), instead of purely histopathologic features. ${ }^{22}$ Three general prognostic groups can be established by dividing grade II into tumors with loss of ATRX expression (often correlating with astrocytoma histology), with an intermediate prognosis between good-prognosis IDH-mutant, 1p/19qcodeleted tumors (oligodendroglioma histology), and poor-prognosis IDH-wild type tumors (primarily associated with glioblastoma or anaplastic astrocytoma histology).

The link between the extent of resection and overall survival in patients with glioblastoma has been established in literature. ${ }^{23,24}$ The role of aggressive surgical management in patients with low-grade gliomas, let alone with multiple foci, remains controversial though. ${ }^{25}$ Surgical paradigms are being challenged, i.e., the resection of at least one lesion followed by adjuvant treatment was associated with a survival advantage (median overall survival 12 months) compared with four months for stereotactic biopsy followed by chemotherapy in one study. ${ }^{26}$ In our opinion, the risks of aggressive surgery (e.g., hemorrhage or neurological deficit) should be weighed against the dubious benefit of debulking surgery. On the other hand, if there is a predominant lesion causing severe mass effect, then a debulking surgery might be discussed in the context of a multidisciplinary team. The role of the tumor's molecular profile (IDH1, 1p/19q codeletion) might aid the decision process in the future. ${ }^{25}$

The role of radiation therapy is also under transformation in the modern era. Historically, whole-brain radiotherapy (WBRT) has been used to treat multifocal/multicentric gliomas. However, WBRT is being questioned, as most failures are traced within the original tumor extent, and high doses cannot be delivered to the entire brain. ${ }^{6,27}$ Despite the numerous foci in our patient, we opted for targeted radiotherapy considering his young age, favorable histology, and the inability to escalate dose with whole-brain radiotherapy. Therefore, he was treated with a VMAT technique and a simultaneous integrated boost to the contrast-enhancing areas. Concurrent with the irradiation, he received temozolomide $75 \mathrm{mg} / \mathrm{m}^{2}$. After the completion of chemoradiation, he continued with adjuvant temozolomide for 12 months.

The omission of whole-brain radiotherapy known to have deleterious cognitive effects, the use of chemotherapy combined with state-of-the-art, dose-escalated, highly focused irradiation, and the presumably favorable biology of the disease are the reasons we believe our patient did exceptionally well.

\section{Conclusion}

In summary, multifocal gliomas are relatively rare, probably underdiagnosed, and therefore under-reported. The role of surgery is limited and stereotactic biopsy is paramount to exclude other pathology and guide treatment planning via molecular characterization. Whole-brain radiotherapy should be abandoned in favor of targeted high dose IMRT/VMAT, allowing safe and effective dose escalation.

\section{Acknowledgments}

None.

\section{Conflicts of interest}

Authors declare that there is no conflict of interest.

\section{References}

1. Porter KR, McCarthy BJ, Freels S, et al. Prevalence estimates for primary brain tumors in the United States by age, gender, behavior, and histology. Neuro Oncol. 2010;12(6):520-527.

2. Mamiya T, Shinji S, Nishizawa T, et al. Malignant Glioma in the Cerebellum Presenting as Multiple Small Lesions. Case Rep Oncol Med. 2019;2019:6725127.

3. Thomas RP, Xu LW, Lober RM, et al. The incidence and significance of multiple lesions in glioblastoma. J Neurooncol. 2013;112(1):91-97.

4. Batzdorf U, Malamud N. The problem of multicentric gliomas. $J$ Neurosurg. 1963;20:122-136.

5. Kyritsis AP, Levin VA, Yung WK, et al. Imaging patterns of multifocal gliomas. Eur J Radiol. 1993;16(3):163-170.

6. Showalter TN, Andrel J, Andrews DW, et al. Multifocal glioblastoma multiforme: prognostic factors and patterns of progression. Int J Radiat Oncol Biol Phys. 2007;69(3):820-824.

7. Larsen J, Hoggard N, McKevitt FM. Imaging in low-grade glioma: a guide for neurologists. Pract Neurol. 2018;18(1):27-34.

8. Kreth FW, Faist M, Rossner R, et al. Supratentorial World Health Organization Grade 2 astrocytomas and oligoastrocytomas. A new pattern of prognostic factors. Cancer. 1997;79(2):370-379.

9. Ginsberg LE, Fuller GN, Hashmi M, et al. The significance of lack of MR contrast enhancement of supratentorial brain tumors in adults: histopathological evaluation of a series. Surg Neurol. 1998;49(4):436440.

10. Shaw E, Arusell R, Scheithauer B, et al. Prospective randomized trial of low- versus high-dose radiation therapy in adults with supratentorial lowgrade glioma: initial report of a North Central Cancer Treatment Group/ Radiation Therapy Oncology Group/Eastern Cooperative Oncology Group study. J Clin Oncol. 2002;20(9):2267-2276.

11. Geurts M, van den Bent MJ. On high-risk, low-grade glioma: What distinguishes high from low? Cancer. 2019;125(2):174-176.

12. Scott JN, Brasher PMA, Sevick RJ, et al. How often are nonenhancing supratentorial gliomas malignant? A population study. Neurology. 2002;59(6):947-949.

13. Ozawa Y, Machida T, Noda M, et al. MRI findings of multiple malignant gliomas: differentiation from multiple metastatic brain tumors. Radiat Med. 1998;16(2):69-74.

14. Rees JH, Smirniotopoulos JG, Jones RV, et al. Glioblastoma multiforme: radiologic-pathologic correlation. Radiographics. 1996;16(6):1413-1438.

15. Wang $\mathrm{P}, \mathrm{Wu} \mathrm{MC}$, Chen SJ, et al. Multicentric gliomas misdiagnosed as metastatic tumors: One case report and literature review. Clinical Oncology and Cancer Research. 2010;7(5):317-321. 
16. Lucchinetti CF, Gavrilova RH, Metz I, et al. Clinical and radiographic spectrum of pathologically confirmed tumefactive multiple sclerosis. Brain. 2008;131(Pt 7):1759-1775.

17. Xia L, Ling S, Wang Z, et al. Tumefactive demyelinating lesions: nine cases and a review of the literature. Neurosurg Rev. 2009;32(2):171-179.

18. Peterson K, Rosenblum MK, Powers JM, et al. Effect of brain irradiation on demyelinating lesions. Neurology. 1993;43(10):2105-2112.

19. Braganza MZ, Kitahara CM, Inskip PD, et al. Ionizing radiation and the risk of brain and central nervous system tumors: a systematic review. Neuro Oncol. 2012;14(11):1316-1324.

20. Pallud J, Audureau E, Blonski M, et al. Epileptic seizures in diffuse lowgrade gliomas in adults. Brain. 2014;137(Pt 2):449-462.

21. van Breemen MSM, Wilms EB, Vecht CJ. Epilepsy in patients with brain tumours: epidemiology, mechanisms, and management. Lancet Neurol. 2007;6(5):421-430

22. Weller M, Tonn JC, Stupp R, et al. European Association for NeuroOncology (EANO) guideline on the diagnosis and treatment of adult astrocytic and oligodendroglial gliomas. Lancet Oncol. 2017;18(6):e315e329.
23. McGirt MJ, Chaichana KL, Gathinji M, et al. Independent association of extent of resection with survival in patients with malignant brain astrocytoma. J Neurosurg. 2009;110(1):156-162.

24. Brown TJ, Brennan MC, Li M, et al. Association of the Extent of Resection With Survival in Glioblastoma: A Systematic Review and Meta-analysis. JAMA Oncol. 2016;2(11):1460-1469.

25. Rudà R, Soffietti R. Extent of surgery in low-grade gliomas: an old question in a new context. Neuro Oncology. 2018;20(1):6-7.

26. di Russo P, Perrini P, Pasqualetti F, et al. Management and outcome of high-grade multicentric gliomas: a contemporary single-institution series and review of the literature. Acta Neurochir. 2013;155(12):2245-2251.

27. Patil CG, Eboli P, Hu J. Management of multifocal and multicentric gliomas. Neurosurg Clin N Am. 2012;23(2):343-350. 